\title{
Asymptotic BEP and SEP of Differential EGC in Correlated Ricean Fading and Non-Gaussian Noise
}

\author{
Ali Nezampour ${ }^{\dagger}$, Amir Nasri ${ }^{\dagger}$, Robert Schober ${ }^{\dagger}$, and Yao $\mathrm{Ma}^{\dagger \dagger}$ \\ ${ }^{\dagger}$ University of British Columbia. E-mail: \{alinezam, amirn, rschober\}@ece.ubc.ca \\ ${ }^{\dagger}$ Iowa State University, E-mail: mayao@iastate.edu
}

\begin{abstract}
In this paper, we study the asymptotic behavior of the bit-error probability (BEP) and symbol-error probability (SEP) of differential $M$-ary phase-shift keying with differential equal gain combining (DEGC) in correlated Ricean fading and non-Gaussian noise, which in our definition also includes interference. We derive simple and easy-to-evaluate asymptotic BEP and SEP expressions which show that at high signalto-noise ratios (SNRs) the performance of DEGC depends on certain moments of the noise and interference impairing the transmission. We provide closed-form expressions for these moments for practically important types of noise such as Gaussian noise, Gaussian mixture noise, and correlated co-channel interference. In addition, we show that the performance loss of DEGC compared to coherent maximum ratio combining (MRC) is always $3 \mathrm{~dB}$ independent of the type of noise if only one diversity branch is available but strongly depends on the type of noise if multiple diversity branches are combined.
\end{abstract}

\section{INTRODUCTION}

In recent years the performance analysis of wireless communication systems impaired by fading and noise has received considerable attention, cf. e.g. [1] and references therein. In particular, for fading channels impaired by additive white Gaussian noise (AWGN), simple closed-form expressions for the symbol error probability (SEP) at high signal-to-noise ratios (SNRs) have been developed, cf. [2]-[5]. These asymptotic expressions are very useful for communication system design as they reveal the effects of the modulation scheme, the diversity combining scheme, and the channel parameters on system performance.

In practice, wireless communication systems are often not only impaired by AWGN, but also by non-Gaussian noise and interference $^{1}$. Examples of non-Gaussian noise include cochannel and adjacent channel interference [6], impulsive noise [7], and ultra-wideband (UWB) interference. Recently, the authors have provided asymptotic SEP results for linear modulation schemes impaired by fading and general non-Gaussian noise assuming coherent equal gain combining (EGC) [8], coherent selection combining (SC) [8], and coherent maximum ratio combining (MRC) [9] at the receiver. Unfortunately, the asymptotic analysis in [8] and [9] is not applicable to differential $M$-ary phase-shift keying ( $M$-PSK) with differential EGC (DEGC) which is often preferred in practice over coherent combining schemes since it does not require channel estimation and phase tracking [1], [2]. We note that unlike coherent combining [4], for DEGC even for impairment

\footnotetext{
${ }^{1}$ To simplify our notation, in the following, "noise" refers to any additive impairment of the received signal, i.e., our definition of noise also includes what is commonly referred to as "interference".
}

by AWGN a general asymptotic performance analysis for correlated fading channels is not available in the literature. Only results for the special case of independent diversity branches can be found in [2], [10].

In this paper, we develop a novel powerful framework for analyzing the bit-error probability (BEP) and SEP of differential $M$-PSK with DEGC in the high SNR regime when the received signal is impaired by correlated Ricean fading and general non-Gaussian noise. In our analysis, the only assumption that is made on the noise is that all of its moments exist. Thus, our results are applicable to a large number of practically relevant scenarios including impairment by AWGN, Gaussian mixture noise, and correlated co-channel interference. The resulting asymptotic BEP and SEP expressions are surprisingly simple and easy to evaluate and only require the calculation of certain noise moments.

The remainder of this paper is organized as follows. In Section II, some definitions and the considered signal model are introduced. In Section III, general expressions for the asymptotic BEP and SEP of differential $M$-PSK with DEGC are derived. The moments required for evaluation of these asymptotic expressions are calculated for several relevant types of noise in Section IV. In Section V, the obtained analytical results are confirmed by simulations, and conclusions are drawn in Section VI.

\section{PRELIMINARIES}

In this section, we introduce some definitions and notations, present the considered signal and channel model, and briefly review the DEGC decision rule.

\section{A. Some Definitions and Notations}

Notation: In this paper, bold lower case letters $\boldsymbol{x}$ and bold upper case letters $\boldsymbol{X}$ denote vectors and matrices, respectively. Furthermore, $\mathcal{E}\{\cdot\}, \operatorname{Pr}\{A\},[\cdot]^{T},(\cdot)^{*},[\cdot]^{H},\|\cdot\|$, and $\operatorname{det}(\cdot)$ denote statistical expectation, the probability of event $A$, transposition, complex conjugation, Hermitian transposition, the $L_{2}$-norm of a vector, and the determinant of a matrix, respectively. In addition, $A \doteq B$ means that $A$ is asymptotically (for high SNR) equal to $B,(2 N-1) ! ! \triangleq 1 \cdot 3 \cdot \ldots \cdot(2 N-1)$, and $(2 N) ! ! \triangleq 2 \cdot 4 \cdot \ldots \cdot 2 N$. Finally, $\Phi(s)=\mathcal{L}\{p(x)\} \triangleq$ $\int_{-\infty}^{\infty} p(x) e^{-s x} \mathrm{~d} x$ denotes the Laplace transform of $p(x)$, and $\boldsymbol{I}_{X}$ and $\mathbf{0}_{X}$ are the $X \times X$ identity matrix and the $X$ dimensional all-zeros column vector, respectively.

Moments: We define the $N$ th moment of the real random variable $(\mathrm{RV})|x|^{2}$ as $M_{x}(N) \triangleq \mathcal{E}\left\{|x|^{2 N}\right\}$, where $x$ is a 
complex RV. Similarly, for a complex random vector variable (RVV) $\boldsymbol{x}$ we define the $N$ th moment of $\|\boldsymbol{x}\|^{2}$ as $M_{\boldsymbol{x}}(N) \triangleq$ $\mathcal{E}\left\{\|\boldsymbol{x}\|^{2 N}\right\}$. We note that $M_{\boldsymbol{x}}(0)=1$ and $M_{\boldsymbol{x}}(1)$ is the sum of the powers of the elements of $\boldsymbol{x}$.

Combining gain and diversity gain: For high SNRs, the SEP in flat fading channels can be approximated by [2], [4]

$$
\mathrm{SEP} \doteq\left(G_{c} \bar{\gamma}\right)^{-G_{d}}
$$

where $\bar{\gamma}$ denotes the average SNR, and $G_{c}$ and $G_{d}$ are referred to as the combining gain ${ }^{2}$ and the diversity gain, respectively.

\section{B. Signal Model}

We assume that the received signal $r_{l}[k]$ in the $l$ th diversity branch in the $k$ th symbol interval can be modeled in equivalent complex baseband representation as

$$
r_{l}[k]=\sqrt{\bar{\gamma}} h_{l}[k] b[k]+n_{l}[k], \quad 1 \leq l \leq L,
$$

where $L, h_{l}[k], b[k]$, and $n_{l}[k]$ denote the number of diversity branches, the fading gain of the $l$ th branch, the transmitted symbol, and the noise in the $l$ th diversity branch, respectively. The $M$-PSK symbol $b[k]$ is obtained by differential encoding $b[k]=a[k] b[k-1]$ from the information bearing differential symbol $a[k]$. Both $b[k]$ and $a[k]$ belong to the same $M-$ PSK constellation $\mathcal{A}$. We assume that the transmitted symbols $b[k] \in \mathcal{A}$ are normalized to $\mathcal{E}\left\{|b[k]|^{2}\right\}=1$. Since the main goal of this paper is to investigate the dependence of the performance of DEGC on the type of noise, we assume that the fading is approximately constant in two successive symbol intervals, i.e., $h_{l}[k]=h_{l}[k-1]=h_{l}, 1 \leq l \leq L$. This assumption is valid if the coherence time of the channel is much larger than the symbol duration, which is true for most practical systems.

Using vector notation, (2) can be rewritten as

$$
\boldsymbol{r}[k]=\sqrt{\bar{\gamma}} \boldsymbol{h} b[k]+\boldsymbol{n}[k],
$$

where $\boldsymbol{r}[k] \triangleq\left[r_{1}[k] r_{2}[k] \ldots r_{L}[k]\right]^{T}, \boldsymbol{h} \triangleq\left[h_{1} h_{2} \ldots h_{L}\right]^{T}$, and $\boldsymbol{n}[k] \triangleq\left[n_{1}[k] n_{2}[k] \ldots n_{L}[k]\right]^{T}$. The channel vector $\boldsymbol{h}$ is assumed to be Gaussian distributed with mean $\boldsymbol{\mu}_{h} \triangleq \mathcal{E}\{\boldsymbol{h}\}$ and covariance matrix $\boldsymbol{C}_{h h} \triangleq \mathcal{E}\left\{\left(\boldsymbol{h}-\boldsymbol{\mu}_{h}\right)\left(\boldsymbol{h}-\boldsymbol{\mu}_{h}\right)^{H}\right\}$. Furthermore, we assume that $\boldsymbol{C}_{h h}$ has full rank $L$ and define the Ricean factor of the $l$ th branch as $K_{l} \triangleq\left|\mu_{l}\right|^{2} / \sigma_{l}^{2}$, where $\mu_{l}$ and $\sigma_{l}^{2}$ denote the $l$ th element of $\boldsymbol{\mu}_{h}$ and the $l$ th main diagonal element of $\boldsymbol{C}_{h h}$, respectively. For convenience we apply the normalization $M_{\boldsymbol{h}}(1)=L$, and we note that for Rayleigh and Ricean fading $\boldsymbol{\mu}_{h}=\mathbf{0}_{L}$ and $\boldsymbol{\mu}_{h} \neq \mathbf{0}_{L}$, respectively.

The noise vector $\boldsymbol{n}[k]$ is independent of $\boldsymbol{h}$ and normalized to $M_{\boldsymbol{n}}(1)=L$. We note that the elements of $\boldsymbol{n}[k]$ may be statistically dependent, non-circular, and non-Gaussian. The only condition that we impose on $\boldsymbol{n}[k]$ is that certain noise moments exist, cf. Section III-A.

\footnotetext{
${ }^{2}$ The combining gain is also often referred to as "coding gain" in the literature, e.g. [4]. We prefer the term "combining gain" as channel coding is not applied here.
}

\section{Differential Equal Gain Combining (DEGC)}

The DEGC $^{3}$ decision rule can be expressed as [1]

$$
\hat{a}[k]=\underset{\tilde{a}[k] \in \mathcal{A}}{\operatorname{argmin}}\left\{\|\boldsymbol{r}[k]-\tilde{a}[k] \boldsymbol{r}[k-1]\|^{2}\right\},
$$

where $\hat{a}[k]$ and $\tilde{a}[k]$ are the estimated symbol and a hypothetical symbol, respectively. We note that the decision rule in (4) may be suboptimum in non-Gaussian noise. However, optimizing the DEGC decision rule for the underlying type of noise is difficult in practice, since the noise statistics are typically not known at the receiver and may change with time. Estimating and tracking these statistics is computationally expensive. Therefore, (4) is usually used regardless of the type of noise that impairs the received signal.

\section{Asymptotic Performance AnAlysis}

In this section, we develop asymptotic expressions for the BEP and SEP of DEGC, investigate the diversity and combining gains of DEGC in non-Gaussian noise, and compare the results for DEGC with those obtained in [9] for MRC. However, first we derive an asymptotic result for the pairwise error probability (PEP).

\section{A. Asymptotic Pairwise Error Probability (PEP)}

The PEP is defined as the probability of detecting $\bar{a}[k]$ while $a[k]$ is transmitted, where $a[k], \bar{a}[k] \in \mathcal{A}$ and $a[k] \neq \bar{a}[k]$. Using the signal model and the decision rule described in the previous section, the PEP can be expressed as

$$
P_{e}(d)=\operatorname{Pr}\left\{\|\sqrt{\bar{\gamma}} \boldsymbol{h} e+\overline{\boldsymbol{n}}\|^{2}<\|\tilde{\boldsymbol{n}}\|^{2}\right\},
$$

where

$$
\begin{aligned}
& \overline{\boldsymbol{n}} \triangleq \boldsymbol{n}[k]-\bar{a}[k] \boldsymbol{n}[k-1] \\
& \tilde{\boldsymbol{n}} \triangleq \boldsymbol{n}[k]-a[k] \boldsymbol{n}[k-1]
\end{aligned}
$$

denote two noise vectors and $e \triangleq(a[k]-\bar{a}[k]) b[k-1]$ is a complex scalar with $d^{2} \triangleq|e|^{2}$. Note that if the marginal probability density functions (pdfs) $p_{n_{l}}\left(n_{l}\right), 1 \leq l \leq L$, of all components of $\boldsymbol{n}[k-1]$ are circular [11], i.e., $p_{n_{l}}\left(n_{l}\right)=$ $p_{n_{l}}\left(e^{j \varphi} n_{l}\right)$ for all $\varphi \in[-\pi, \pi)$, and $\boldsymbol{n}[k]$ and $\boldsymbol{n}[k-1]$ are statistically independent, $a[k]$ has no influence on the distribution of $\tilde{\boldsymbol{n}}$ and $\tilde{\boldsymbol{n}} \triangleq \boldsymbol{n}[k]-\boldsymbol{n}[k-1]$ may be used instead of the definition in (7). A similar statement applies to $\overline{\boldsymbol{n}}$.

Based on (5) we can express the conditional PEP as

$$
P_{e}(d \mid \overline{\boldsymbol{n}}, \tilde{\boldsymbol{n}})=\int_{0}^{\|\tilde{\boldsymbol{n}}\|^{2}} p_{\Delta}(x) \mathrm{d} x,
$$

where $p_{\Delta}(x)$ denotes the pdf of $\Delta \triangleq\|\boldsymbol{u}\|^{2}$ with $\boldsymbol{u} \triangleq \sqrt{\bar{\gamma}} \boldsymbol{h} e+$ $\overline{\boldsymbol{n}}$. Conditioned on $\overline{\boldsymbol{n}}, \boldsymbol{u}$ is a Gaussian random vector with mean $\boldsymbol{\mu}_{u} \triangleq \mathcal{E}\{\boldsymbol{u} \mid \overline{\boldsymbol{n}}\}=\sqrt{\bar{\gamma}}$ e $\boldsymbol{\mu}_{h}+\overline{\boldsymbol{n}}$ and covariance matrix $\boldsymbol{C}_{u u} \triangleq \mathcal{E}\left\{\boldsymbol{u} \boldsymbol{u}^{H} \mid \overline{\boldsymbol{n}}\right\}=\bar{\gamma}|e|^{2} \boldsymbol{C}_{h h}$. Therefore, the Laplace

${ }^{3}$ DEGC is also referred to as "differentially coherent" EGC and "postdetection" EGC in the literature, cf. e.g. [1]. 
transform $\Phi_{\Delta}(s) \triangleq \mathcal{E}\left\{e^{-s \Delta}\right\}$ of $p_{\Delta}(x)$ can be expressed as [12]

$$
\Phi_{\Delta}(s)=\frac{\exp \left(-s \boldsymbol{\mu}_{u}^{H}\left(\boldsymbol{I}_{L}+s \boldsymbol{C}_{u u}\right)^{-1} \boldsymbol{\mu}_{u}\right)}{\operatorname{det}\left(\boldsymbol{I}_{L}+s \boldsymbol{C}_{u u}\right)} .
$$

For full rank fading correlation matrices $\boldsymbol{C}_{h h}$ and $\bar{\gamma} \rightarrow \infty$, (9) can be simplified to

$$
\Phi_{\Delta}(s) \doteq \frac{\exp \left(-\left[\boldsymbol{\mu}_{h}+\frac{\overline{\boldsymbol{n}}}{e \sqrt{\bar{\gamma}}}\right]^{H} \boldsymbol{C}_{h h}^{-1}\left[\boldsymbol{\mu}_{h}+\frac{\overline{\boldsymbol{n}}}{e \sqrt{\bar{\gamma}}}\right]\right)}{\operatorname{det}\left(\boldsymbol{C}_{h h}\right) d^{2 L} \bar{\gamma}^{L} s^{L}} .
$$

An asymptotic expression for $p_{\Delta}(x)$ can now be easily obtained by applying the inverse Laplace transform to (10). This result can then be used in (8) to obtain the asymptotic conditional PEP

$$
\begin{aligned}
& P_{e}(d \mid \overline{\boldsymbol{n}}, \tilde{\boldsymbol{n}}) \doteq \\
& \quad \frac{\exp \left(-\left[\boldsymbol{\mu}_{h}+\frac{\overline{\boldsymbol{n}}}{e \sqrt{\bar{\gamma}}}\right]^{H} \boldsymbol{C}_{h h}^{-1}\left[\boldsymbol{\mu}_{h}+\frac{\overline{\boldsymbol{n}}}{e \sqrt{\bar{\gamma}}}\right]\right)}{L ! \operatorname{det}\left(\boldsymbol{C}_{h h}\right) d^{2 L} \bar{\gamma}^{L}}\|\tilde{\boldsymbol{n}}\|^{2 L} .
\end{aligned}
$$

Using the expansion $\exp (x)=\sum_{k=0}^{\infty} x^{k} / k$ !, we can rewrite the exponential function in (11) as

$$
\begin{array}{r}
\exp \left(-\left[\boldsymbol{\mu}_{h}+\overline{\boldsymbol{n}} /(e \sqrt{\bar{\gamma}})\right]^{H} \boldsymbol{C}_{h h}^{-1}\left[\boldsymbol{\mu}_{h}+\overline{\boldsymbol{n}} /(e \sqrt{\bar{\gamma}})\right]\right)= \\
\exp \left(-\boldsymbol{\mu}_{h}^{H} \boldsymbol{C}_{h h}^{-1} \boldsymbol{\mu}_{h}\right)(1+f(\overline{\boldsymbol{n}}) / \bar{\gamma}),
\end{array}
$$

where $f(\overline{\boldsymbol{n}})$ is implicitly defined in (12). Furthermore, $f(\overline{\boldsymbol{n}})$ can be written as a sum of products of the form $C_{\kappa_{1}, \nu_{1}, \cdots, \kappa_{L}, \nu_{L}} \bar{n}_{1}^{\kappa_{1}}\left(\bar{n}_{1}^{*}\right)^{\nu_{1}} \bar{n}_{2}^{\kappa_{2}}\left(\bar{n}_{2}^{*}\right)^{\nu_{2}} \cdots \bar{n}_{L}^{\kappa_{L}}\left(\bar{n}_{L}^{*}\right)^{\nu_{L}}$, where $C_{\kappa_{1}, \nu_{1}, \cdots, \kappa_{L}, \nu_{L}}$ are coefficients that are non-increasing in $\bar{\gamma}, \bar{n}_{l}, 1 \leq l \leq L$, denote the elements of $\overline{\boldsymbol{n}}$, and $\kappa_{l} \geq 0$ and $\nu_{l} \geq 0$ are integers. Assuming now that all individual and joint moments of the elements of $\overline{\boldsymbol{n}}$ and $\tilde{\boldsymbol{n}}$ exist (i.e., $\mathcal{E}\left\{\bar{n}_{1}^{\kappa_{1,1}}\left(\bar{n}_{1}^{*}\right)^{\nu_{1,1}} \tilde{n}_{1}^{\kappa_{2,1}}\left(\tilde{n}_{1}^{*}\right)^{\nu_{2,1}} \cdots \bar{n}_{L}^{\kappa_{1, L}}\left(\bar{n}_{L}^{*}\right)^{\nu_{1, L}} \tilde{n}_{L}^{\kappa_{2, L}}\left(\tilde{n}_{L}^{*}\right)^{\nu_{2, L}}\right\}$ $<\infty$, where $\tilde{n}_{l}$ are the elements of $\tilde{\boldsymbol{n}}$, and $\nu_{1, l} \geq 0, \nu_{2, l} \geq 0$, $\kappa_{1, l} \geq 0$, and $\kappa_{2, l} \geq 0,1 \leq l \leq L$ ), when $\bar{\gamma} \rightarrow \infty$, from (11) and (12) we obtain for the (unconditional) PEP the simple asymptotic expression

$$
P_{e}(d)=\mathcal{E}\left\{P_{e}(d \mid \overline{\boldsymbol{n}}, \tilde{\boldsymbol{n}})\right\} \doteq \frac{p_{h} M_{\tilde{\boldsymbol{n}}}(L)}{L ! d^{2 L}} \bar{\gamma}^{-L}
$$

with

$$
p_{h} \triangleq \frac{\exp \left(-\boldsymbol{\mu}_{h}^{H} \boldsymbol{C}_{h h}^{-1} \boldsymbol{\mu}_{h}\right)}{\operatorname{det}\left(\boldsymbol{C}_{h h}\right)} .
$$

From (13) we observe that $\overline{\boldsymbol{n}}$ has no influence on the asymptotic PEP. Furthermore, $\tilde{\boldsymbol{n}}$ affects the PEP via $M_{\tilde{\boldsymbol{n}}}(L)$, i.e., only the number of diversity branches $L$ determines which moment of $\|\tilde{\boldsymbol{n}}\|^{2}$ is relevant for the PEP, but the mean $\boldsymbol{\mu}_{h}$ and the correlation matrix $\boldsymbol{C}_{h h}$ of $\boldsymbol{h}$ have no influence in this regard.

\section{B. Asymptotic SEP and BEP}

At high SNRs, the SEP will be dominated by the PEP of the nearest-neighbor signal points of $\mathcal{A}$. Thus, from (13) we obtain

$$
\mathrm{SEP} \doteq \frac{\beta_{M} p_{h} M_{\tilde{\boldsymbol{n}}}(L)}{L ! d_{M}^{2 L}} \bar{\gamma}^{-L}
$$

where $d_{M}=2 \sin (\pi / M)$ denotes the minimum Euclidean distance of $\mathcal{A}$ and $\beta_{M}$ is the average number of minimumdistance neighbors $\left(\beta_{M}=1\right.$ for $M=2$ (BPSK) and $\beta_{M}=2$ for $M \geq 4$ ). Assuming that Gray mapping is used for nonbinary modulations, the asymptotic BEP can be obtained from the corresponding SEP as [2]

$$
\mathrm{BEP} \doteq \frac{\mathrm{SEP}}{\log _{2}(M)}
$$

From (15) and (16) the SEP and BEP of differential $M_{-}$ PSK with DEGC can be easily calculated as long as closedform expressions for the moments $M_{\tilde{\boldsymbol{n}}}(L)$ are available. The calculation of these moments for practically relevant types of noise and interference is addressed in Section IV.

\section{Diversity and Combining Gain}

A comparison of (15) with (1) shows immediately that the diversity gain of DEGC is $G_{d}=L$, independent of the type of noise. Furthermore, on a logarithmic scale, the combining gain can be expressed as

$$
\begin{aligned}
G_{c}= & \frac{10}{L} \log _{10}(L !)+\frac{10}{L} \log _{10}\left(\frac{d_{M}^{2 L}}{\beta_{M}}\right) \\
& -\frac{10}{L} \log _{10}\left(p_{h}\right)-\frac{10}{L} \log _{10}\left(M_{\tilde{n}}(L)\right) .
\end{aligned}
$$

The second, the third, and the fourth term of (17) show the dependence of $G_{c}$ on the modulation scheme, the channel statistics, and the noise statistics, respectively. Eq. (17) reveals that for a given $L$, the modulation scheme, the channel statistics, and the noise statistics independently contribute to the combining gain.

Furthermore, an important result is drawn from (17) for the special case of $L=1$. In this case, $M_{\tilde{n}}(1)=\mathcal{E}\{\| n[k]-$ $\left.\left.a[k] n[k-1]\right|^{2}\right\}=2 M_{n}(1)-2 \Re\left\{\mathcal{E}\left\{a^{*}[k]\right\} \mathcal{E}\left\{n[k] n^{*}[k-\right.\right.$ $1]\}\}=2$ holds for all types of noise because $\mathcal{E}\left\{a^{*}[k]\right\}=0$ for $M$-PSK. Therefore, (17) shows that for $L=1$ the asymptotic error rate performance of DEGC is independent of the type of noise.

\section{Comparison with MRC}

A comparison of (15) with the results in [9] shows that (15) is also valid for $M$-PSK (without differential encoding) with coherent MRC if $M_{\tilde{\boldsymbol{n}}}(L)$ is replaced by $M_{\boldsymbol{n}}(L)$. This shows that DEGC and MRC have the same diversity gain $G_{d}=L$. However, DEGC suffers from a combining gain loss compared to coherent MRC. This loss is given by

$$
\Delta G_{c}=\frac{10}{L} \log _{10}\left(\frac{M_{\tilde{n}}(L)}{M_{\boldsymbol{n}}(L)}\right) .
$$

Eq. (18) shows that the combining gain loss of DEGC compared to coherent MRC depends on the number of diversity branches $L$ and the noise statistics. However, it is independent of the fading statistics $\boldsymbol{\mu}_{h}$ and $\boldsymbol{C}_{h h}$.

For the special case of $L=1, M_{\boldsymbol{n}}(1)=1$ is always valid. Thus, since it was shown in the previous section that $M_{\tilde{\boldsymbol{n}}}(1)=$ 2 , we obtain $\Delta G_{c}=3 \mathrm{~dB}$ for $L=1$ independent of the type of noise. It will be shown in Section IV that $\Delta G_{c}$ does depend on the type of noise for $L>1$. 


\section{Calculation of Noise Moments}

In this section, we derive closed-form expressions for $M_{\tilde{\boldsymbol{n}}}(L)$ for AWGN, spatially independent noise, spatially dependent Gaussian mixture noise, and correlated co-channel interference. To facilitate the exposition in this section, we have collected the moments of a few relevant scalar RVs and RVVs in Table I.

\section{A. I.I.D. Gaussian Noise (AWGN)}

Since impairment by independent identically distributed (i.i.d.) Gaussian noise is often assumed in the literature, it is instructive to consider this case also for our asymptotic analysis of DEGC. Here, $\|\boldsymbol{n}[k]\|^{2}$ and $\|\tilde{\boldsymbol{n}}\|^{2}$ are both chisquare distributed RVs with $2 L$ degrees of freedom and it is straightforward to show that $M_{\tilde{\boldsymbol{n}}}(L)$ can be expressed as

$$
M_{\tilde{\boldsymbol{n}}}(L)=2^{L} M_{\boldsymbol{n}}(L),
$$

where $M_{\boldsymbol{n}}(L)$ is given in Table I (i.i.d. Gaussian RVV). Therefore, according to (18), for AWGN the asymptotic performance loss of DEGC compared to coherent MRC is $\Delta G_{c}=3 \mathrm{~dB}$, independent of the number of diversity branches and independent of $\boldsymbol{\mu}_{h}$ and $\boldsymbol{C}_{h h}$. This $3 \mathrm{~dB}$ loss is a well-known result for the special case of BPSK transmission over i.i.d. Rayleigh fading channels, cf. [2, Section 14.4.1], but seems to be new for general (correlated) Ricean fading channels. For BPSK modulation, i.i.d. Rayleigh fading, and AWGN it can also be verified that (15) is equivalent to [2, Eq. (14.4-28)].

\section{B. Spatially Independent Noise}

For many practically relevant scenarios, the noise components in different diversity branches are mutually independent. In this case, we can use the multinomial expansion [13] to calculate $M_{\boldsymbol{n}}(L)$ as

$$
M_{\boldsymbol{n}}(L)=\sum_{k_{1}+\ldots+k_{L}=L}\left(\begin{array}{c}
L \\
k_{1}, \ldots, k_{L}
\end{array}\right) M_{n_{1}}\left(k_{1}\right) \cdot \ldots \cdot M_{n_{L}}\left(k_{L}\right),
$$

where the $M_{n_{l}}\left(k_{l}\right)$ denote the moments of the components $n_{l}[k], 1 \leq l \leq L$, of $\boldsymbol{n}[k]$. The moment $M_{\tilde{\boldsymbol{n}}}(L)$ of $\tilde{\boldsymbol{n}}$ can also be obtained from (20) by replacing $M_{n_{l}}\left(k_{l}\right)$ with $M_{\tilde{n}_{l}}\left(k_{l}\right)$, $1 \leq l \leq L$. If we assume furthermore that $n_{l}[k]$ is circular (cf. Section III-A) and temporally independent (i.e., $v \triangleq n_{l}[k]$ and $w \triangleq n_{l}[k-1]$ are independent), the scalar moments $M_{\tilde{n}_{l}}(N)$ can be calculated as

$$
M_{\tilde{n}_{l}}(N)=\sum_{k_{1}+2 k_{2}+k_{3}=N} \frac{N !}{k_{1} !\left(k_{2} !\right)^{2} k_{3} !} M_{v}\left(k_{1}+k_{2}\right) M_{w}\left(k_{2}+k_{3}\right) .
$$

To illustrate the application of (20) and (21), we consider spatially independent Gaussian mixture noise. Gaussian mixture RVs are used to model the combined effect of Gaussian background noise and man-made, impulsive noise [7]. The pdf of scalar Gaussian mixture noise with $I$ terms is given by

$$
p_{n}(n)=\sum_{k=1}^{I} \frac{c_{k}}{\pi \sigma_{k}^{2}} \exp \left(-\frac{|n|^{2}}{\sigma_{k}^{2}}\right)
$$

where $c_{k}>0, \sum_{k=1}^{I} c_{k}=1$, and $\sigma_{k}^{2}, 1 \leq k \leq I$, are constants. Special cases of Gaussian mixture noise include Middelton's Class-A noise $(I \rightarrow \infty)$ [14] and $\epsilon$-mixture noise $(I=2)$. Specifically, $\epsilon$-mixture noise with variance $\sigma_{n}^{2}$ is characterized by $c_{1}=1-\epsilon, c_{2}=\epsilon, \sigma_{1}^{2}=\sigma_{n}^{2} /(1-\epsilon+\kappa \epsilon)$, $\sigma_{2}^{2}=\kappa \sigma_{n}^{2} /(1-\epsilon+\kappa \epsilon), 0 \leq \epsilon<1$, and $\kappa>1$. The moments $M_{n}(N)$ of scalar Gaussian mixture RVs are given in Table I.

Assuming that all diversity branches are affected by spatially (and temporally) independent Gaussian mixture noise ${ }^{4}$, $M_{\tilde{\boldsymbol{n}}}(L)$ can be easily calculated using (20), (21), and $M_{n}(N)$ from Table I. For example, for $L=2$ and temporally i.i.d. $\epsilon$-mixture noise, we obtain from (18)

$$
\Delta G_{c}=3 \mathrm{~dB}+5 \log _{10}\left(\frac{\left(1-\epsilon+\epsilon \kappa^{2}\right)+2(1-\epsilon+\epsilon \kappa)^{2}}{2\left(1-\epsilon+\epsilon \kappa^{2}\right)+(1-\epsilon+\epsilon \kappa)^{2}}\right)
$$

which yields $\Delta G_{c}=3 \mathrm{~dB}$ for $\epsilon=0$ (as expected for AWGN) and $\Delta G_{c}<3 \mathrm{~dB}$ for $\epsilon>0$ (impulsive noise). For example, for $\epsilon=0.25$ (23) yields $\Delta G_{c}=2.4 \mathrm{~dB}$ and $2.2 \mathrm{~dB}$ for $\kappa=10$ and $\kappa=50$, respectively. Therefore, (23) clearly shows the noise dependence of the combining gain loss of DEGC compared to MRC.

\section{Spatially Dependent Gaussian Mixture Noise}

The pdf of spatially dependent Gaussian mixture (vector) noise ("Model I" in [7]) is given by

$$
p_{\boldsymbol{n}}(\boldsymbol{n})=\sum_{k=1}^{I} \frac{c_{k}}{\pi^{L} \sigma_{k}^{2 L}} \exp \left(-\frac{\|\boldsymbol{n}\|^{2}}{\sigma_{k}^{2}}\right)
$$

where $c_{k}>0, \sum_{k=1}^{I} c_{k}=1$, and $\sigma_{k}^{2}, 1 \leq k \leq I$, are constants. This is an appropriate model for impulsive noise if the physical process causing the impulsive behavior affects all antennas simultaneously [7]. The corresponding moment $M_{\boldsymbol{n}}(L)$ is given in Table I.

Assuming temporally independent, spatially dependent Gaussian mixture noise, it is easy to show that $\tilde{\boldsymbol{n}}$ is also a Gaussian mixture RVV whose pdf can be obtained from (24) by replacing $I, c_{k}$, and $\sigma_{k}^{2}$ with $\tilde{I} \triangleq I(I+1) / 2, \tilde{c}_{k}$, and $\tilde{\sigma}_{k}^{2}$, respectively. The latter two parameters are defined as $\tilde{c}_{k} \triangleq c_{k}^{2}$ and $\tilde{\sigma}_{k}^{2} \triangleq 2 \sigma_{k}^{2}$ for $1 \leq k \leq I$, and $\tilde{c}_{k} \triangleq 2 c_{i} c_{j}$ and $\tilde{\sigma}_{k}^{2} \triangleq \sigma_{i}^{2}+\sigma_{j}^{2}$ for $I+1 \leq k \leq \overline{\tilde{I}}, 1 \leq i \leq I, 1 \leq j \leq I, i \neq j$. With these definitions, $M_{\tilde{\boldsymbol{n}}}(L)$ can also be easily obtained from Table I and the combining gain loss of DEGC compared to coherent MRC can be expressed as

$$
\Delta G_{c}=\frac{10}{L} \log _{10}\left(\frac{\sum_{k=1}^{\tilde{I}} \tilde{c}_{k} \tilde{\sigma}_{k}^{2 L}}{\sum_{k=1}^{I} c_{k} \sigma_{k}^{2 L}}\right)
$$

which for $\epsilon$-mixture noise can be simplified to

$$
\begin{aligned}
& \Delta G_{c}= \\
& \frac{10}{L} \log _{10}\left(\frac{(1-\epsilon)^{2} 2^{L}+2 \epsilon(1-\epsilon)(\kappa+1)^{L}+\epsilon^{2}(2 \kappa)^{L}}{1-\epsilon+\epsilon \kappa^{L}}\right)(26)
\end{aligned}
$$

${ }^{4}$ This noise model is referred to as "Model II" in [7]. It is an appropriate model for impulsive noise if the phenomenon causing the impulsive behavior affects the antennas independently, see [7] for a detailed discussion. 
TABLE I

MOMENTS OF BASIC RVS $\boldsymbol{n}$ AND RVVS $\boldsymbol{n}$ INTRODUCED IN SECTION IV.

\begin{tabular}{|c|l|}
\hline Noise Model & Moments \\
\hline \hline Gaussian Mixture RV & $M_{n}(N)=N ! \sum_{k=1}^{I} c_{k} \sigma_{k}^{2 N}$ \\
\hline$M_{i}$-ary Interference & $M_{n}(N)=\frac{1}{M_{i}^{k_{u}-k_{l}+1} \sum_{n_{0} \in \mathcal{S}}\left|n_{0}\right|^{2 N}}$ \\
\hline I.I.D. Gaussian RVV & $M_{\boldsymbol{n}}(L)=\frac{(2 L-1) !}{(L-1) !} \sigma_{n}^{2 L}$ \\
\hline $\begin{array}{c}\text { Correlated } \\
\text { Gaussian RVV }\end{array}$ & $M_{\boldsymbol{n}}(L)=L ! \sum_{k_{1}+\ldots+k_{L}=L} \lambda_{1}^{k_{1}} \cdot \ldots \cdot \lambda_{L}^{k_{L}}$ \\
\hline $\begin{array}{c}\text { Spatially Dependent } \\
\text { Gaussian Mixture RVV }\end{array}$ & $M_{\boldsymbol{n}}(L)=\frac{(2 L-1) !}{(L-1) !} \sum_{k=1}^{I} c_{k} \sigma_{k}^{2 L}$ \\
\hline
\end{tabular}

Again for $\epsilon=0$ (Gaussian case), (26) yields $\Delta G_{c}=3 \mathrm{~dB}$ as expected. However, for $\epsilon>0$ and $\kappa>1$ it can be shown that $\Delta G_{c}<3 \mathrm{~dB}$. For example, for $\epsilon=0.25$ and $\kappa=10$ we obtain from (26) $\Delta G_{c}=3 \mathrm{~dB}, 2.25 \mathrm{~dB}$, and $2.0 \mathrm{~dB}$ for $L=1,2$, and 3 , respectively.

\section{Asynchronous Co-channel Interference}

A single Rayleigh faded asynchronous co-channel interferer can be modeled as

$$
\boldsymbol{n}[k]=\boldsymbol{g} \cdot z[k],
$$

where $\boldsymbol{g}$ is a correlated zero-mean Gaussian RVV with covariance matrix $\boldsymbol{C}_{\boldsymbol{g g}}$ and

$$
z[k]=\sum_{\kappa=k_{l}}^{k_{u}} p(\kappa T+\tau) i[k-\kappa],
$$

where $k_{l}, k_{u}, T, \tau, p(t)$, and $i[k]$ denote the lower limit, the upper limit, the symbol duration, the delay of the interferer compared to the desired user, the overall interference pulse shape, and the $M_{i}$-ary symbols transmitted by the interferer, respectively. $k_{l}$ and $k_{u}$ are appropriately chosen to ensue $p(\kappa T+\tau) \approx 0$ for $\kappa<k_{l}$ and $\kappa>k_{u}$. Since $\boldsymbol{g}$ and $z[k]$ are statistically independent, the moments of $\boldsymbol{n}$ can be calculated as

$$
M_{\boldsymbol{n}}(L) \triangleq \mathcal{E}\left\{\|\boldsymbol{g} z[k]\|^{2 L}\right\}=M_{\boldsymbol{g}}(L) M_{z}(L),
$$

The moment $M_{z}(L)$ is given in Table I $\left(M_{i}\right.$-ary interference), where $\mathcal{S}$ includes all values of $z[k]$ for the $M_{i}^{k_{u}-k_{l}+1}$ possible combinations of $i[k-\kappa], k_{l} \leq \kappa \leq k_{u} . M_{\boldsymbol{g}}(L)$ is also given in Table I (correlated Gaussian RVV) in terms of the eigenvalues $\lambda_{i}, 1 \leq i \leq L$, of $\boldsymbol{C}_{\boldsymbol{g g}}$.

The relevant noise term for DEGC is given by $\tilde{\boldsymbol{n}}=\boldsymbol{g} \tilde{z}$, where $\tilde{z} \triangleq z[k]-a[k] z[k-1]$ can be modeled as in (28) if $p(\kappa T+\tau)$ and $i[k]$ are replaced with appropriately defined effective coefficients $\tilde{p}(\kappa T+\tau)$ and effective interference symbols $\tilde{i}[k]$, respectively. Therefore, $M_{\tilde{\boldsymbol{n}}}(L)=M_{\boldsymbol{g}}(L) M_{\tilde{z}}(L)$ can also be easily obtained using the results in Table I.

As an example, we consider the special case of a single synchronous co-channel interferer, where $\tilde{z}$ can be simplified to $\tilde{z}=i[k]-a[k] i[k-1]$. Assuming that the desired user and the interferer use the same $M$-PSK constellation, i.e., $M_{i}=$ $M, M_{\tilde{z}}(L)$ can be equivalently calculated from $\tilde{z}=i[k]-i[k-$ $1]$, which yields $M_{\tilde{z}}(L)=\frac{1}{M} 2^{L} \sum_{m=0}^{M-1}[1-\cos (2 \pi m / M)]^{L}$.
Therefore, since $M_{z}(L)=1$ for $M$-PSK, the performance loss of DEGC compared to MRC is given by

$$
\Delta G_{c}=3 \mathrm{~dB}+\frac{10}{L} \log _{10}\left(\frac{1}{M} \sum_{m=0}^{M-1}\left[1-\cos \left(\frac{2 \pi}{M} m\right)\right]^{L}\right) .
$$

Eq. (30) reveals that the performance loss suffered by DEGC in correlated co-channel interference depends only on $L$ and the adopted $M$-PSK constellation. For example, for BPSK $\Delta G_{c}=3(2 L-1) / L \mathrm{~dB}$, whereas for 4-PSK $\Delta G_{c}=3(1-$ $1 / L) \mathrm{dB}+10 \log \left(\sqrt[L]{2^{L-1}+1}\right)$. For both BPSK and 4-PSK, we obtain $\Delta G_{c}=3 \mathrm{~dB}$ for $L=1$ and $\Delta G_{c}=6 \mathrm{~dB}$ for $L \gg 1$. For $M \gg 1$ the sum in (30) can be approximated by an integral. This leads to

$$
\Delta G_{c}=6 \mathrm{~dB}-10 \log _{10}\left(\sqrt[L]{\frac{(2 L) ! !}{(2 L-1) ! !}}\right)
$$

which yields $\Delta G_{c}=3 \mathrm{~dB}, 3.9 \mathrm{~dB}$, and $4.3 \mathrm{~dB}$ for $L=1$, 2 , and 3, respectively, and approaches $6 \mathrm{~dB}$ as $L$ increases. From these considerations we conjecture that for a single synchronous $M$-PSK co-channel interferer and any constellation size $M$ the asymptotic performance loss of DEGC compared to $\mathrm{MRC}$ is between $3 \mathrm{~dB}$ and $6 \mathrm{~dB}$, where the higher value is approached as $L$ increases.

\section{NUMERICAL RESUlTS AND DisCUSSIONS}

In this section, we verify the derived analytical expressions for the asymptotic BEP and SEP of differential $M$-PSK with DEGC for several practically relevant cases with computer simulations ${ }^{5}$. For comparison we also include the BEPs and SEPs of $M$-PSK with coherent MRC [9].

Fig. 1 shows the SEP of 4-PSK for DEGC and MRC in i.i.d. Rayleigh fading $(L=2)$ and impairment by, respectively, i.i.d. Gaussian noise (AWGN) and i.i.d. spatially independent $\epsilon$-mixture noise with $\epsilon=0.25$ and $\kappa=10,50$. The simulation results nicely confirm the derived analytical asymptotic results. Furthermore, we observe that the asymptotic performance loss of DEGC compared to MRC is $\Delta G_{c}=3 \mathrm{~dB}$ for AWGN, and $\Delta G_{c}=2.4 \mathrm{~dB}$ and $2.2 \mathrm{~dB}$ for $\epsilon-$ mixture noise with $\kappa=10$ and $\kappa=50$, respectively. This is in perfect agreement with (23).

In Fig. 2, we consider the BEP of 8-PSK with DEGC and MRC for $L=1,2$, and 3 over an i.i.d. Ricean fading channel ( $K=3 \mathrm{~dB}$ ) impaired by spatially dependent $\epsilon$-mixture noise $(\epsilon=0.25, \kappa=10)$. Again, the simulation results nicely confirm our asymptotic analysis. Furthermore, Fig. 2 shows that the asymptotic performance loss of DEGC compared to MRC is, respectively, $3 \mathrm{~dB}, 2.3 \mathrm{~dB}$, and $2 \mathrm{~dB}$ for $L=1,2$, and 3 , which is in perfect agreement with the results obtained from (26).

In Fig. 3, we show the SEP of 16-PSK with DEGC and MRC for correlated Ricean fading $(K=3 \mathrm{~dB})$ and impairment

\footnotetext{
${ }^{5}$ Since, in this paper, we refer to any additive impairment as "noise", we use the term "SNR" even if the received signal is only impaired by what is traditionally referred to as "interference".
} 


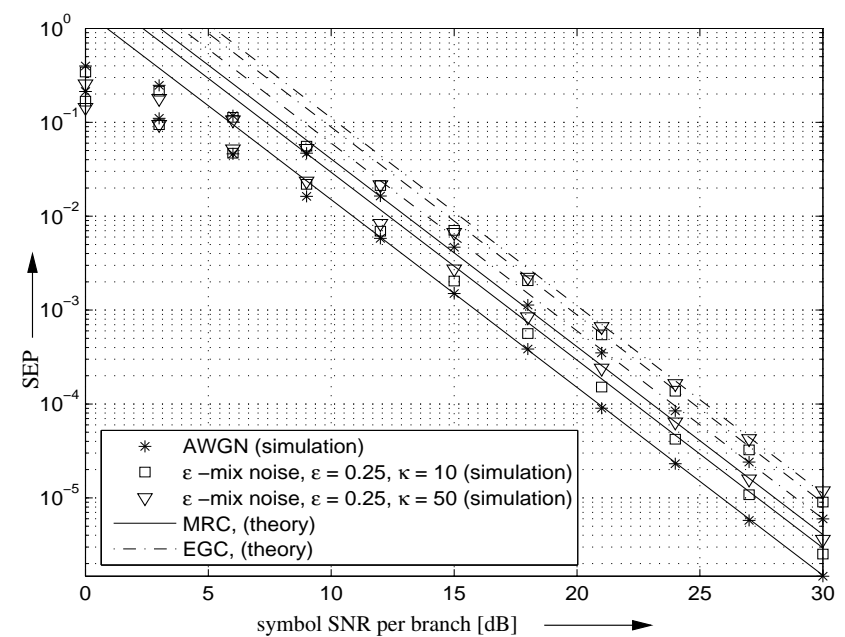

Fig. 1. SEP of 4-PSK vs. symbol SNR per branch for DEGC and MRC over an i.i.d. Rayleigh fading channel $(L=2)$ impaired by AWGN, and i.i.d. spatially independent $\epsilon$-mixture noise with $\epsilon=0.25$ and $\kappa=10$ and $\kappa=50$, respectively. Markers: Simulated SEP. Lines: Asymptotic SEP.

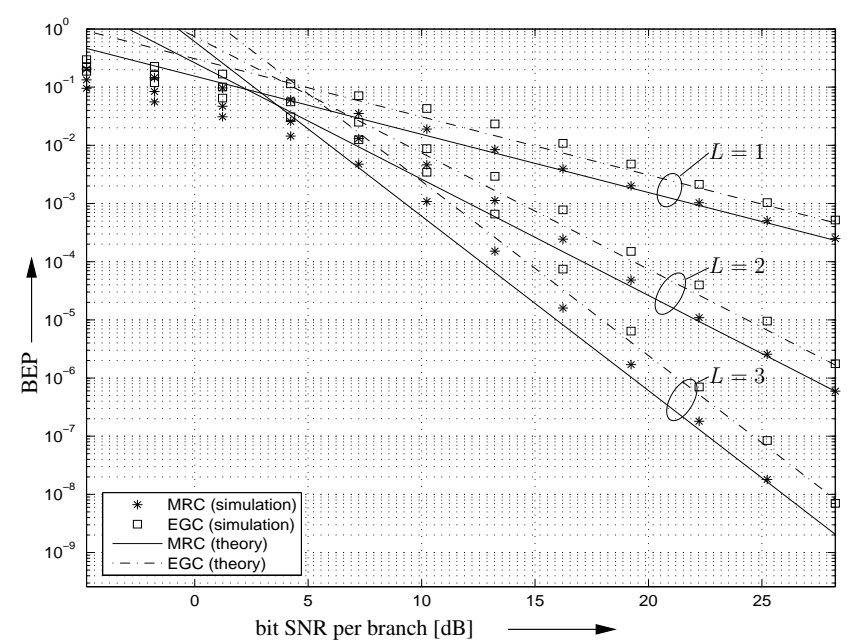

Fig. 2. BEP of 8-PSK vs. bit SNR per branch for DEGC and MRC over an i.i.d. Ricean fading channel with Ricean factor $K=3 \mathrm{~dB}$ and spatially dependent $\epsilon$-mixture noise $(\epsilon=0.25, \kappa=10)$. Markers: Simulated BEP. Lines: Asymptotic BEP.

by a correlated Rayleigh faded synchronous 16-PSK cochannel interferer. For $L=3$ the correlation matrices $\boldsymbol{C}_{h h}$ and $\boldsymbol{C}_{g g}$ of the desired user and the interferer are respectively, Toeplitz matrices with $\left[\begin{array}{lll}1 & \alpha & \alpha^{2}\end{array}\right]$ and $\left[\begin{array}{lll}1 & \rho & \rho^{2}\end{array}\right]$ as the first row, where $\alpha=\rho=0.6$. For $L=2$ Toeplitz matrices with first rows $\left[\begin{array}{ll}1 & \alpha\end{array}\right]$ and $\left[\begin{array}{ll}1 & \rho\end{array}\right]$ and the same values for $\alpha$ and $\rho$ were used. We observe from Fig. 3 that for the considered type of interference, the performance loss of DEGC compared to MRC is $3 \mathrm{~dB}, 3.9 \mathrm{~dB}$, and $4.3 \mathrm{~dB}$ for $L=1,2$, and 3, respectively, which is in perfect agreement with the values obtained from (31).

\section{CONCLUSION}

In this paper, we have presented simple and insightful asymptotic BEP and SEP expressions for differential $M$-PSK with DEGC in correlated Ricean fading and non-Gaussian noise and interference. These asymptotic expressions are easy to evaluate and only require the calculation of certain noise moments. From our asymptotic analysis we draw the following

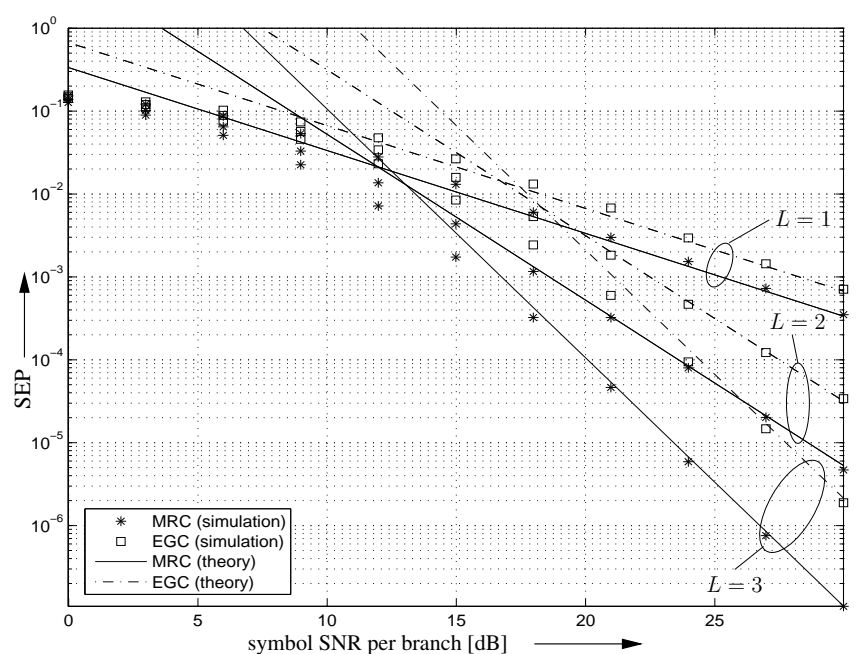

Fig. 3. SEP of 16-PSK vs. symbol SNR per branch for DEGC and MRC over correlated Ricean fading channels $(K=3 \mathrm{~dB}, \alpha=0.6)$ with correlated Rayleigh faded co-channel interference $(\rho=0.6)$. Markers: Simulated SEP. Lines: Asymptotic SEP.

conclusions: (1) For $L=1$ diversity branch the asymptotic performance loss $\Delta G_{c}$ of DEGC compared to MRC is 3 $\mathrm{dB}$ independent of the type of noise. (2) For impairment by AWGN $\Delta G_{c}$ is equal to $3 \mathrm{~dB}$ for all $L$. (3) For non-Gaussian noise and interference and $L>1$ the loss $\Delta G_{c}$ is in general different from $3 \mathrm{~dB}$. In particular, for both spatially dependent and independent $\epsilon$-mixture noise $\Delta G_{c} \leq 3 \mathrm{~dB}$, whereas for for correlated synchronous $M$-PSK co-channel interference $\Delta G_{c} \geq 3 \mathrm{~dB}$.

\section{REFERENCES}

[1] M.K. Simon and M.-S. Alouini. Digital Communication over Fading Channels. Wiley, Hoboken, New Jersey, 2005.

[2] J.G. Proakis. Digital Communications. McGraw-Hill, New York, forth edition, 2001 .

[3] H. Abdel-Ghaffar and S. Pasupathy. Asymptotic Performance of $M$-ary and Binary Signals Over Multipath/Multichannel Rayleigh and Ricean Fading. IEEE Trans. Commun., COM-43:2721-2731, November 1995.

[4] Z. Wang and G.B. Giannakis. A Simple and General Parameterization Quantifying Performance in Fading Channels. IEEE Trans. Commun., COM-51:1389-1398, August 2003.

[5] Z. Du, J. Chen, and N. Beaulieu. Asymptotic BER Performance of OFDM in Frequency-Selective Nakagami-m Channels. In Proceedings of the IEEE Vehicular Technology Conference (VTC), pages 612-615, September 2004.

[6] A. Giorgetti and M. Chiani. Influence of Fading on the Gaussian Approximation for BPSK and QPSK with Asynchronous Cochannel Approximation for BPSK and QPSK with Asynchronous Cochannel

[7] C. Tepedelenlioglu and P. Gao. On Diversity Reception Over Fading Channels with Impulsive Noise. IEEE Trans. Veh. Technol., 54:20372047, November 2005.

[8] A. Nasri, R. Schober, and Y. Ma. Unified Asymptotic Analysis of Linearly Modulated Signals in Fading, Non-Gaussian Noise, and Interference. Revised paper submitted to the IEEE Trans. Commun., October 2006

[9] A. Nezampour, A. Nasri, R. Schober, and Y. Ma. Asymptotic BEP and SEP of MRC in Correlated Ricean Fading and Non-Gaussian Noise. Submitted to the IEEE Vehicular Technology Conference (VTC), Baltimore, February 2007.

[10] Y. Ma, Z. Wang, and S. Pasupathy. Asymptotic Performance of HybridSelection/Maximal-Ratio Combining over Fading Channels. IEEE Trans. Commun., COM-54:770-777, May 2006.

[11] B. Picinbono. On Circularity. IEEE Trans. Signal Processing, 42:34733482, December 1994

[12] M. Schwartz, W. Bennett, and S. Stein. Communication Systems and Techniques. McGraw-Hill, New York, 1966.

[13] M. Abramowitz and I. Stegun. Handbook of Mathematical Functions. Dover Publications, Inc., New York, 1970.

[14] D. Middleton. Statistical-physical Models of Man-made Radio Noise Parts I and II. U.S. Dept. Commerce Office Telecommun., April 1974 and 1976. 